\title{
Valoración del riesgo cardiovascular de pacientes en Hemodiálisis
}

\author{
Accésit Premio de Investigación en Enfermería Nefrológica Janssen-Cilag
}

\section{Cristina Mendías Benítez* - Antonio Abujas Ruiz* - Juan Luis Chaín de la Bastida*** - Rafaela Ponce Martín**}

* Unidad Uronefrológica. Área de Diálisis. Hospitales Universitarios Virgen del Rocío. Sevilla. ** Unidad de Hemodiálisis Clínica Santa Isabel. Sevilla. *** Hospital Universitario Virgen Macarena. Sevilla

\section{Resumen}

Las complicaciones cardiovasculares son la principal causa de enfermedad renal crónica, y a su vez uno de los principales factores de riesgo cardiovascular. Por otro lado, la enfermedad cardiovascular está íntimamente relacionada con la obesidad central o androide.

Nuestro objetivo es determinar los factores de riesgo cardiovascular presentes en nuestros pacientes en tratamiento sustitutivo de hemodiálisis para elaborar una hoja de seguimiento que nos sirva de apoyo para valorar la progresión o regresión de los factores de riesgo.

Se diseñó un estudio descriptivo transversal prospectivo y abierto, la población de referencia se determinó por una muestra de los pacientes en tratamiento hemodialítico en nuestros centros. Analizados los 14 factores de riesgo cardiovascular presentes en el estudio, encontramos una media de factores presentes de $5,11 \pm 2,15$. La población estudiada presentaba: valores altos en cuanto a sobrepeso, uno de cada cuatro pacientes del estudio presentaba Síndrome Metabólico, niveles mayores de troponina basal en pacientes con más tiempo en hemodiálisis, encontramos una variedad entre $0 \mathrm{y}$

Correspondencia:

Cristina Mendías Benítez

Área de Diálisis

Hospitales Universitarios Virgen del Rocío. Sevilla

Av. Manuel Siurot, s/n

41013 Sevilla

Correo electrónico: mendiascris@hotmail.com
9 factores de riesgo presentes de los 14 analizados en el estudio, no encontramos correlación entre el tiempo en hemodiálisis y aumento de los factores de riesgo o entre la presencia de síndrome metabólico y el tiempo en hemodiálisis.

\section{PALABRAS CLAVE:}

- RIESGO CARDIOVASCULAR

- SINDROME METABÓLICO

- INDICE DE MASA CORPORAL

- INDICE TOBILLO BRAZO

- PERIMETRO ABDOMINAL

- ESTUDIO MULTICENTRICO

\section{Assessment of cardiovascular risk in haemodialysis patients}

\section{Abstract}

Cardiovascular complications are the main cause of the Chronic Kidney Disease and also one of the main cardiovascular risk factors. On the other hand, a cardiovascular disease is directly related to android or central obesity. Our goal is to define cardiovascular risk factors on our patients receiving haemodialysis therapy, in order to provide a follow-up form as a support to estimate the progression or regression of risk factors. 
A transversal descriptive study, prospective and opened, has been elaborated. Reference population has been determined by a simple of our patients in haemodialysis therapy of our centres. Having analyzed the 14 cardiovascular risk factors we present in the study, the average factors founded are $5.11 \pm 2,157$.

Population to study showed: high level of overweight, Metabolic Syndrome (1 in 4 patients), Metabolic Syndrome, higher level of basal troponin in haemodialysis patients treated for a long period of time. It has been found an oscillation from 0 to 9 risk factors out of the 14 studied ones. Neither a correlation between duration of haemodialysis treatment and increase of cardiovascular risk factors either between the appearing of Metabolic Syndrome and the duration of haemodialysis has been found.

\section{PALABRAS CLAVE: \\ - CARDIOVASCULAR RISK \\ - METABOLIC SYNDROME \\ - BODY MASS INDEX \\ - ANKLE / ARM INDEX \\ - WAIST CIRCUMFERENCE \\ - MULTICENTER STUDY}

Dentro de este factor, actualmente, se usan criterios de medida del perímetro de la cintura de acuerdo con el sexo, para definir el componente de masa corporal que contribuye a factor de riesgo cardiovascular (FRCV). Incluso las bases para el uso del perímetro abdominal (PA) se cimientan en el hecho de que las medidas globales de la obesidad, como el IMC, son indicadores poco sensibles del riesgo de complicaciones metabólicas o cardiovascular de la obesidad, sobre todo comparadas con las determinaciones específicas de adiposidad central 0 visceral. Sin embargo, el perímetro de la cintura se correlaciona muy bien con el contenido de adiposidad intraabdominal, y es considerado un método sencillo y fiable como parámetro de valoración del riesgo cardiovascular de los pacientes.

A parte de los factores clásicos de RCV, dependientes del estilo de vida (dieta, sedentarismo, estrés, tabaco, alcohol), diabetes, se incluye el Síndrome Metabólico (SM), que se puede definir como el conjunto de alteraciones metabólicas y cardiovasculares que aparecen relacionadas con un estado de insulinorresistencia. Recientes propuestas sugieren que el SM sea considerado como una condición de riesgo intermedio de enfermedad cardiovascular (riesgo del 10-20\% de desarrollar complicación cardiovascular proyectada a 10 años). Existen varios criterios para la valoración del SM, pero todos coinciden en que se establece el SM si existe la presencia de tres 0 más factores.

La presencia de Enfermería ante este planteamiento queda extensamente justificada en la necesidad de desarrollar la prevención, detección precoz y tratamiento, quizás mejor, modificación de los factores de riesgo. No debemos limitarnos a la determinación del perímetro abdominal, glucemia basal o índice tobillobrazo de un paciente, si no ser el punto de partida en una educación que lleve a una mejora de los diferentes factores que germinen en el paciente desarrollando un estilo de vida cardiosaludable ${ }^{3}$.

\section{Objetivos}

Nuestros objetivos fueron determinar los factores de $\mathrm{RCV}$ presentes en nuestros pacientes en tratamiento sustitutivo de hemodiálisis para elaborar una hoja de seguimiento que nos sirva de apoyo para valorar la progresión o regresión de los factores de riesgo. 


\section{Metodología}

Se diseñó un estudio descriptivo transversal prospectivo y abierto. La población de referencia se determinó por los pacientes en tratamiento hemodialítico en nuestros centros. Los datos fueron obtenidos durante el primer trimestre del 2009 y, para su análisis, descriptivo, estratificado y de frecuencias y correlaciones, utilizamos el paquete estadístico SPSS 15.
El estudio consta de 73 pacientes, todos en programa de hemodiálisis. Formaban el grupo de pacientes crónicos con diálisis en el hospital de referencia 11 pacientes $y$ 62 en un centro periférico, de ellos 39 eran varones y 34 mujeres, con edades comprendidas entre $34-82$ años, cuya media es de 64,5 y una desviación de $\pm 13,05$.

Se realizó un cuestionario (ANEXO I) para valorar los diferentes factores dependientes del estilo de vida.

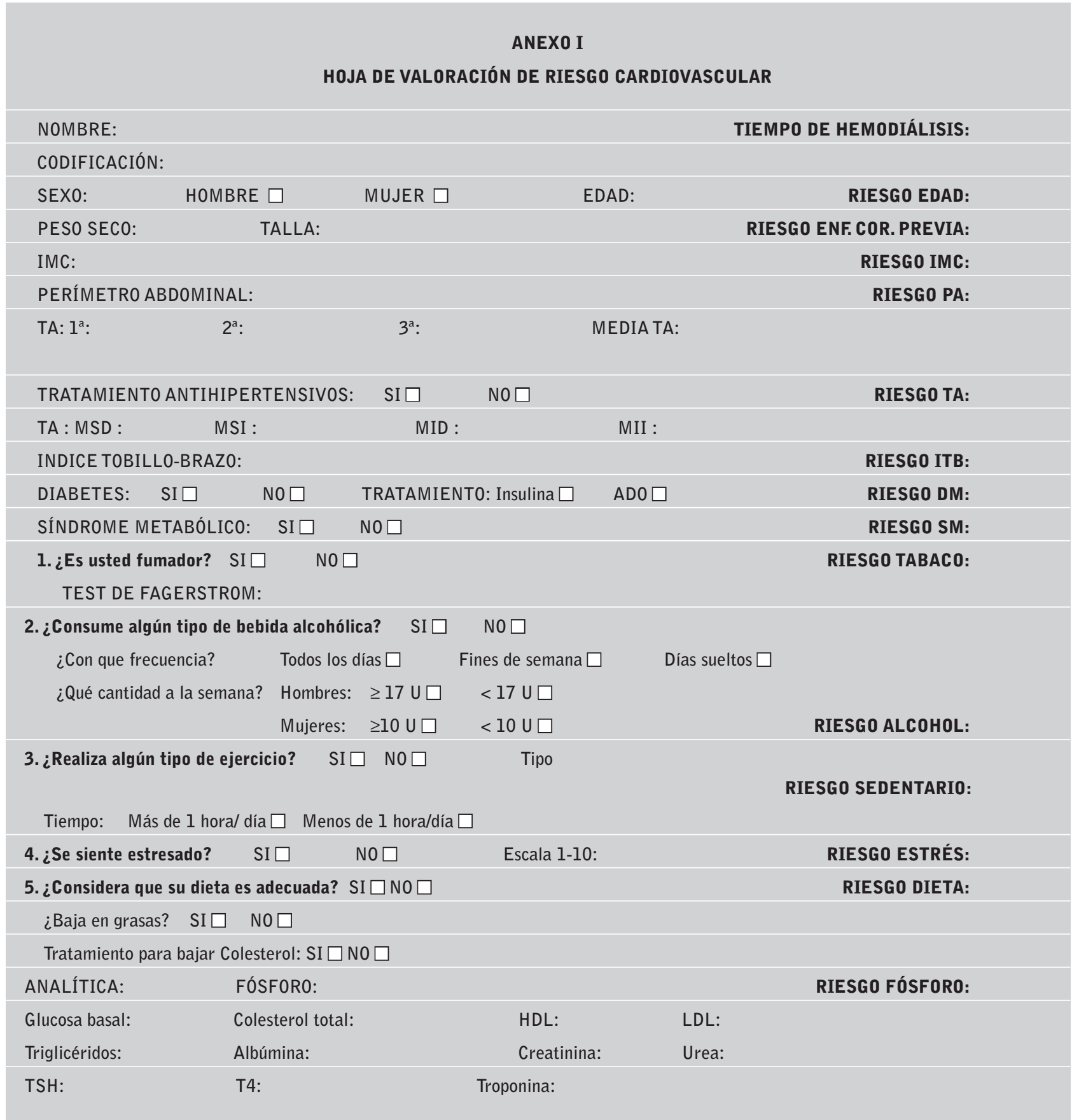


Como pruebas complementarias analizamos: glucosa basal, urea, creatinina, albúmina, colesterol total, HDL, LDL, triglicéridos, fósforo, troponina y hormonas tiroideas (TSH, T4). Los criterios de valoración para los diferentes parámetros utilizados fueron:

- Edad: en hombres el factor de riesgo es para mayores de 55 años y en mujeres mayores de 65 años

- Tiempo en Hemodiálisis en meses

- Enfermedad coronaria previa

- IMC

- Perímetro abdominal

- Tensión Arterial: medida en mmHg. con esfingomanómetro manual o electrónico, en el brazo dominante de pacientes con acceso vascular de catéter y en el brazo contrario en caso de acceso de FAVI, con una media de tres tensiones tomada prehemodiálisis durante los tres días de la semana de su programa habitual. Según la escala de estratificación ${ }^{4}$, nuestros pacientes, al tener afectación de órgano diana (trastornos clínicos asociados), estarían en Alto riesgo o Riesgo Muy Alto.

- Índice tobillo/brazo: medida en mmHg. con esfingomanómetro de la tensión arterial sistólica en los diferentes miembros: MSD, MSI, MID, MII, teniendo en cuenta pacientes portadores de FAVI y calculando el cociente de la presión sistólica mayor del miembro inferior entre presión sistólica mayor de miembro superior.

- Diabetes.

- Tabaco. Como dato extra, analizamos la dependencia del tabaco a los pacientes que fuman mediante el test de Fagerstrom.

- Alcohol. Para que se considere factor de riesgo consumir algún tipo de bebida alcohólica debe de sobrepasar una cantidad, en hombres mayor o igual de 170 gramos de alcohol ó 17 unidades a la semana y 100 gramos ó 10 unidades en la mujer.

- Síndrome metabólico: Existen varios criterios para la valoración del Síndrome Metabólico, pero todos coinciden en que se establece el síndrome Metabólico si existe la presencia de tres o más factores. Analizaremos el SM según Criterios del Nacional Colesterol Education Program-Adult Treatment Panel III (tabla 1).
- Ejercicio: estratificación en persona activa, persona parcialmente activa y persona sedentaria.

- Estrés: sensación subjetiva de estrés del paciente, estratificada mediante escala de likert del uno al diez.

- Dieta.

- Fósforo: consideramos riesgo cardiovascular un fósforo superior de $4,5 \mathrm{mg} / \mathrm{dl}$.

\begin{tabular}{|l|l|l|}
\hline $\begin{array}{l}\text { PERÍMETRO } \\
\text { ABDOMINAL }\end{array}$ & Hombres: $>102 \mathrm{~cm}$. & Mujeres: $>88 \mathrm{~cm}$. \\
\hline $\begin{array}{l}\text { TRIGLICÉRIDOS } \\
\text { COLESTEROL- HDL }\end{array}$ & $\geq 150 \mathrm{mg} / \mathrm{dl}$. & \\
\hline $\begin{array}{l}\text { PRESIÓN } \\
\text { ARTERIAL }\end{array}$ & $\geq 130 / 85 \mathrm{mmHg}$. & \\
\hline $\begin{array}{l}\text { GLUCEMIAS EN } \\
\text { AYUNAS }\end{array}$ & $\geq 110 \mathrm{mg} / \mathrm{dl}$. & \\
\hline
\end{tabular}

Tabla 1. Síndrome Metabólico según Criterios del Nacional Colesterol Education Program - Adult Treatment Panel III (NCEP. ATP .III)

\section{Resultados}

El $76,9 \%$ de los hombres es $>55$ años y el $35,2 \%$ de las mujeres es $>65$ años. El 58,9\% de la población había presentado algún episodio coronario previo. Un $64,1 \%$ de los hombres frente a un $52,9 \%$ de las mujeres. En cuanto al IMC, en los hombres no hay ninguno con Peso Insuficiente, el 35,8\% presentan peso normal, el 20,5\% Sobrepeso Grado I, el 20,5\% Sobrepeso Grado II, el 17,9\% Obesidad tipo I, y 7,6\% Obesidad tipo II. Las mujeres presentan el 2,9\% Peso Insuficiente, el 35,2\% Normo peso, el 17,6\% Sobrepeso Grado I, el 17,6\% Sobrepeso Grado II, el 20,5\% Obesidad tipo I, el 2,9\% Obesidad tipo II y el 2,9\% Obesidad tipo III.

El Perímetro Abdominal de los hombres en un $35,8 \%$ es mayor de $102 \mathrm{~cm}$., en las mujeres en un $41,1 \%$ es mayor de $88 \mathrm{~cm}$.

El $43,5 \%$ de los hombres presenta tensión arterial Normal el 28,2\% Normal-Alta el 20,5\%, Hipertensión Grado I y el 7,6\% Hipertensión Grado II. Las mujeres presentan en un 44,1\% Tensión arterial Normal, el 
20,5\% Normal-Alta, el 29,4\% Hipertensión Grado I, el 5,8\% Hipertensión Grado II. El 48,7\% de los hombres toman algún tratamiento antihipertensivo frente al $52,9 \%$ de las mujeres. El 5,1\% de los pacientes masculinos fue imposible la medición de la tensión arterial en miembros inferiores, y el 14,7\% de las mujeres, por lo que lo incluimos en el porcentaje de pacientes que presentaban el factor de riesgo cardiovascular.

Al considerar el índice Tobillo/brazo se observa que el $38,4 \%$ de los hombres presenta Calcificación Arterial y el $17,9 \%$ Arteriopatía Ligera-Moderada. Las mujeres presentan un 29,4\% Calcificación Arterial, $32,3 \%$ normalidad y Arteriopatía Ligera-Moderada el $2,9 \%$.

El 23,3\% de la población es diabética. Un 30,7\% de los hombres frente al $11,7 \%$ de las mujeres. El 23,0\% de los hombres presentan Síndrome Metabólico frente al $44,1 \%$ de las mujeres.

Son fumadores el $7,6 \%$ de los hombres; respecto a la dependencia del tabaco (Test de Fagerstrom) el $66,6 \%$ que no pre sentan Dependencia y el $33,3 \%$ una Dependencia Moderada. Las mujeres en un $11,7 \%$ son fumadoras, con un Test de Fagerstrom de $75 \%$ No Dependientes y un $25 \%$ con Dependencia Moderada.

El $46,1 \%$ de los hombres consumen algún tipo de bebida alcohólica, el $16,6 \%$ en una cantidad $\geq 17$ uu. y el $83,3 \%<17$ uu. Las mujeres consumen bebida alcohólica en un $41,1 \%$, siendo un $28,5 \%$ en una cantidad $\geq 10$ uu. y el $71,4 \%<10$ uu. Los hombres son en un $31,5 \%$ personas activas, un $31,5 \%$ personas parcialmente activas y un $37 \%$ personas sedentarias. Las mujeres son un $14,7 \%$ personas activas, un $38,2 \%$ personas parcialmente activas y un $47 \%$ sedentarias.

El 33,3\% de los hombres se sienten estresados, valorando en una Escala del 1-10, una media de 5,4 puntos. Un $50 \%$ de las mujeres se sienten estresadas, con una media de 7,1 puntos.

Los hombres consideran en un $76,9 \%$ que tienen una Dieta Adecuada y las mujeres lo consideran en un $79,4 \%$. El 35,8\% de los hombres tienen un Fósforo $\geq 4,5 \mathrm{mg} / \mathrm{dl}$ frente al $55,8 \%$ de las mujeres.
Analizados los 14 factores de Riesgo Cardiovascular presentes en el estudio, encontramos una media de factores presentes de 5,11 $\pm 2,157$ riesgo (figura $1)$. Las correlaciones realizadas fueron no paramétricas mediante Rho de Spearman, encontrando los siguientes resultados: hubo correlación significativa de 0,05 entre el perímetro abdominal y enfermedad coronaria previa y la significación fue de 0,01 entre el tiempo en meses en hemodiálisis y los valores de troponina T y entre IMC y Perímetro Abdominal. Igual significación encontramos en el Índice Tobillo/ Brazo y el tiempo de los pacientes en diálisis.

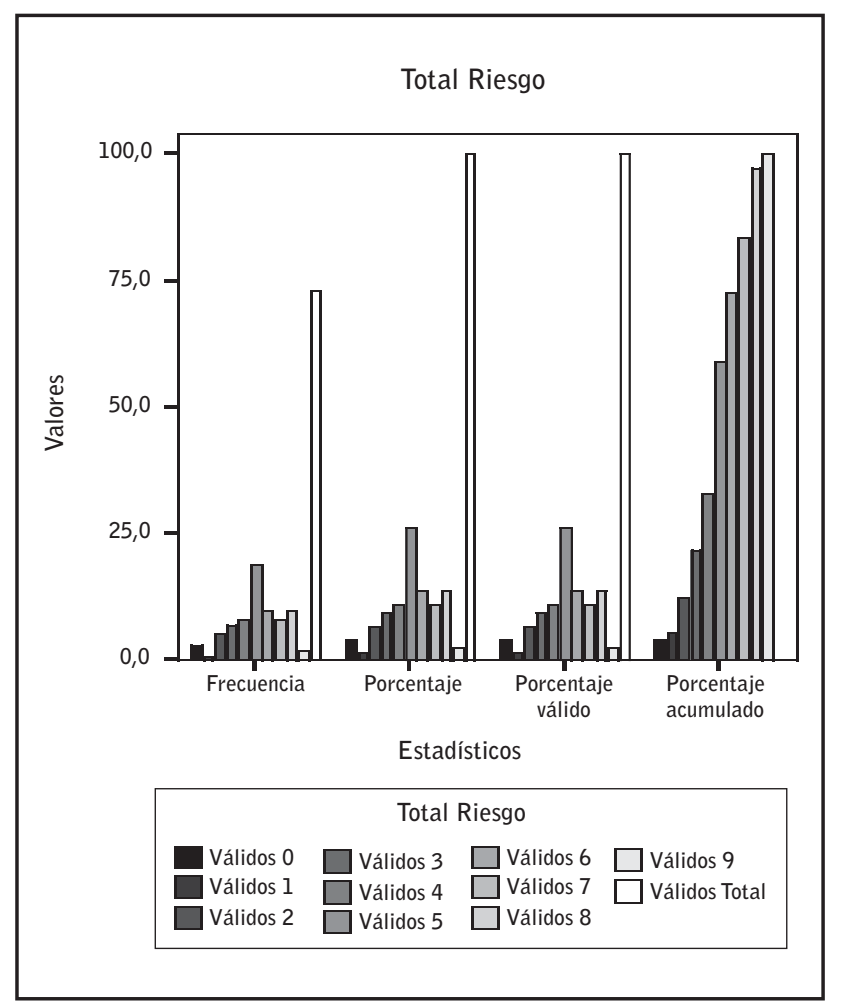

Figura 1. Factores de riesgo presentes.

\section{Discusión y conclusiones}

Tanto en la determinación de dieta adecuada como en la de niveles subjetivos de estrés utilizamos lo que consideramos posteriormente como escalas muy subjetivas, si bien, hemos considerado, por la importancia de la misma en la ERC, que la dieta necesita una revisión a parte para su valoración y desarrollo. En el caso de la detección de niveles de estrés estamos buscando un cuestionario, dentro de la gran variedad existente, que más se adapte a las posibilidades del paciente (por 
ejemplo, pasamos una "escala de estrés percibido" a pacientes de nivel cultural medio-alto que reconocieron dificultades para interpretar los diferentes ítems). Muchos pacientes no diferenciaban el estrés percibido con el grado de ansiedad-depresión.

La población estudiada presentaba una variedad de entre 0 y 9 factores de riesgo, basándonos en el total, encontramos que tenemos una población activa-parcialmente activa, en los que cerca de 6 de cada 10 pacientes han presentado un episodio de enfermedad coronaria previa. Uno de cada cuatro pacientes del estudio presentaba Síndrome Metabólico. Hay un mayor riesgo de arteriopatía en los pacientes estudiados conforme aumentan los meses con tratamiento sustitutivo.

La población estudiada presentaba unos valores altos en cuanto sobrepeso; no así referente a obesidad o peso insuficiente, tanto en la población femenina como en la masculina. Proporciones similares encontramos en referencia al PA. Mantenemos una población diabética cercana a la de la población de diálisis en general ${ }^{5-7}$, aunque en los hombres es más significativo que en las mujeres. En pacientes que presentan episodios previos de enfermedad coronaria se ha encontrado un mayor perímetro abdominal.

Detectamos unos niveles mayores de troponina basal en pacientes con más tiempo en hemodiálisis. Sin embargo, no encontramos correlación entre tiempo en HD y aumento de los FRCV o entre la presencia de SM y el tiempo en HD. La mayoría de los pacientes presentan un adecuado control hipertensivo.

\section{Agradecimientos}

Nuestro agradecimiento por su colaboración a la Dra. Guillermina Fernández. Jefe de Servicio de la Unidad de Hemodiálisis Clínica Santa Isabel, al Dr Juan J. Castilla Jiménez, Jefe de Sección de Nefrología del Hospital Universitario Virgen del Rocío y a las enfermeras y enfermeros, y auxiliares de enfermería de ambos servicios que han colaborado, siendo su único interés el bien del paciente.

\section{Bibliografía}

1. K/DOQI clinical practice guidelines for chronic kidney disease: evaluation, classification, and stratification. Part 7. Stratification of risk for progression of kidney disease and development of cardiovascular disease.

2. Aguirre Rodríguez, JC et al. A propósito de un caso en HTA: Diabetes y Síndrome Metabólico. Decisiones Clínicas en Atención Primaria. Madrid 2006.

3. Iborra Moltó C, Roca Alonso M, Picó Mira L, Huéscar Massó B, López Roig S. Síndrome Metabólico en hemodiálisis . Actuación enfermera. Rev. Soc. Esp. Enferm. Nefrol 2008, 11 (2): 89-94.

4. K/DOQI Clinical Practise Guidelines for Chronic Kidney Disease: Evaluation, Classification and Stratification. Am J. Kidney Dis 2003:39 (Suppl1): S1-S89.

5. Guías SEN "Riñón y Enfermedad Cardiovascular". Nefrología 2004: Vol. 24.

6. Brotonsa C, Royo-Bordonada MA, Alvarez-Salad L et all. Adaptación de la guía europea de prevención cardiovascular. Nefrología. 2004. 24(4): 312-328.

7. J. Portolés, JM López Gómez, P. Aljama y AM Tato, en nombre del Grupo de estudio MAR. Riesgo Cardiovascular en Hemodiálisis en España: prevalencia, pautas de actuación y objetivos (estudio MAR). Nefrología, Vol. XXV. Número 3. 2005. 297-305. 